\title{
Fatigue Damage Analysis of Composite Insulator Cor Rod/sheath Interface
}

\author{
Xinze Zhao, Senming Xi, Meiyun Zhao ${ }^{a}$, Wenyun PENG, Seng Tian, Wei Li \\ Hubei Key Laboratory of Hydroelectric Machinery Design \& Maintenance, China Three Gorges University, Yichang, 443002
}

\begin{abstract}
A self-made fatigue testing machine was used to simulate the damage process of the insulator interface under working conditions and the damage degree is evaluated by the compressive shear test. The improved interface adhesion model reflects the interface debonding process under different fatigue loads by introducing the damage coefficient $\chi$, migration coefficient $\alpha$ and the debonding coefficient $\mu$. The relationship of fatigue loads and interface damage is established by the peeling force, the unit interface separation work and the peeling time. The test demonstrates that the interface damage near the bottom of the insulator is worse than that near the middle part. The result of this analysis will be beneficial in easily finding damaged places of the insulator, and so as to reduce the power breakdown.
\end{abstract}

\section{Introduction}

The composite insulator is an insulating device widely used in high voltage transmission line, mainly consisting of metal fittings, core rod, and housing. The core rod consists of fiber-glass and epoxy resin, and it is the main load-bearing part. The main material of the sheath is silicone rubber. Cohesive interface between core rod and sheath runs throughout two ends of metal fittings, which is the key part of the insulation [1-2]. Due to the effects of the overhead line weight, the wind load and other factors, the force of composite insulator in operation is very complicated [3-5]. The mechanical fatigue, environment temperature changing, atmosphere corrosion, high field strength and so on, all lead to core $\mathrm{rod} / \mathrm{sheath}$ interface deteriorative failure [6-8], and eventually may result to the serious accidents [9-11]. It is of great significance to analyze the fatigue damage of the bonding interface of the core rod and sheath under different loads, which can reduce the failure of insulators. At home and abroad, the interface is evaluated by measuring the interfacial bonding strength or detecting interface defects. You et al. [12] introduced a method to test the interfacial bonding strength of two sides of the flexible matrix with a 180-degree angle stripping. Zhang et al. [13] used the interface constitutive model and 90degree peeling test to analyze the bonding strength of the anisotropic conductive adhesive film from theoretically and experimentally, and used the damage factors to illustrate the changes of interface bonding strength under high temperature and humidity loading. The interfacial bonding strength was divided into several grades by the anatomical grading test method, and the improved water diffusion experiment could discover the quality defect of the composite insulator that traditional methods could not find [14-15]. Duan et al. [16] used the core-sheath compressive shear test to evaluated the interfacial adhesion strengths of the composite insulator. Most of them used the confirmatory test to measure the interfacial quality between sheath and core, while the fatigue parameters are not considered, it is difficult to indicate the influence about degree of damage to the interfacial adhesion of the insulator.

In this paper, we combined the constitutive model of bonding interface proposed by Needleman. by means of theoretical analysis and compressive shear test, the peeling process of core rod/sheath bonding interface under the different fatigue conditions was analyzed and the change of residual bond strength of the interface was described with the unit area separation work and the peeling time. It provides a reference to the research of the mechanical fatigue failure of composite insulators.

\section{Test}

\subsection{Core rod/ sheath interface and parameters}

FXBW4-110/100 suspension composite insulator which is used in $110 \mathrm{kV}$ transmission line, was selected as the test object. Under normal circumstances the interface between sheath and core rod should be tightly bonded. However, due to the mechanical fatigue produced by selfweight of transmission line, swing and torsion under wind load [17-18], the interface of composite insulator could have air gaps and even crack. Under the working conditions the force of the composite insulator core $\mathrm{rod} / \mathrm{sheath}$ interface is shown in Figure 1.

\footnotetext{
${ }^{a}$ Corresponding author: zhaomeiyun@ctgu.edu.cn
} 


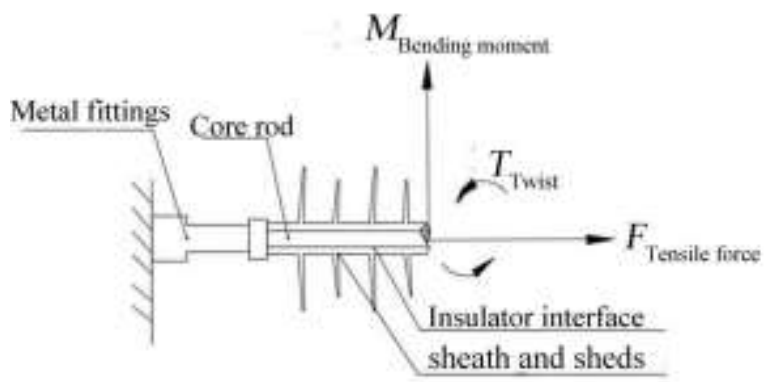

Figure 1. Force diagram of composite insulators on core/sheath interface

\subsection{Fatigue test}

In order to simulate the fatigue failure process of core $\mathrm{rod} / \mathrm{sheath}$ interface of composite insulators, dynamic fatigue test was carried out on a self-made fatigue device until the insulator being ruptured, as shown in Figure 2 [19]. The test machine can put different frequency pull, bend, torsion load on the composite insulator to simulate the process of fatigue under the wire weight, and wind load including swing and twist. Insulator fatigue test system is shown in Figure 3, and the test design is shown in Table 1.

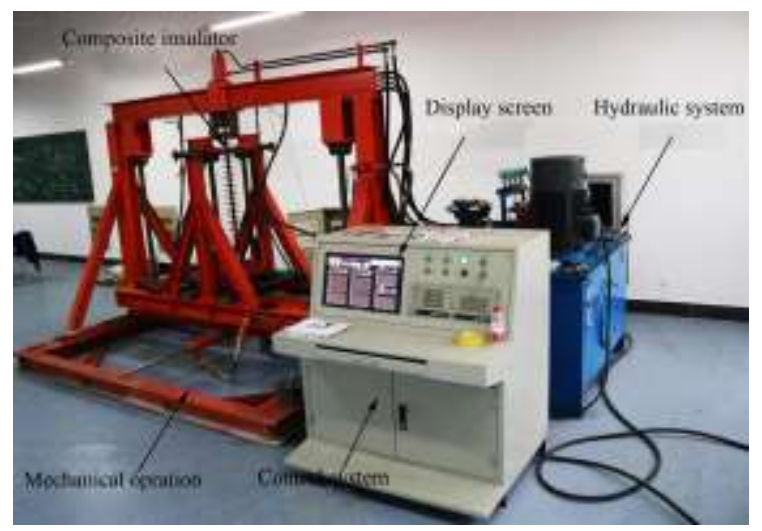

Figure 2. Fatigue test machine

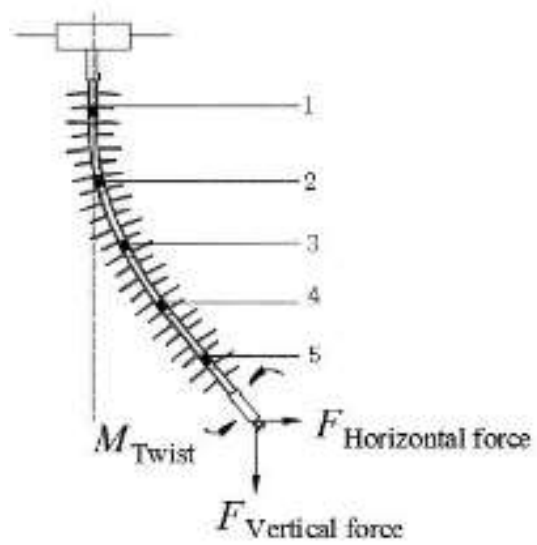

Figure 3. Composite insulator force diagram
Table. 1 Fatigue test scheme of composite insulators

\begin{tabular}{|c|c|c|c|}
\hline Test groups & $\begin{array}{c}\text { Tensile force } \\
\boldsymbol{F} / \mathbf{M P a}\end{array}$ & $\begin{array}{c}\text { Swing } \\
\text { amplitude } \\
\boldsymbol{L} / \mathbf{m m}\end{array}$ & $\begin{array}{c}\text { Twist angle } \\
\boldsymbol{\alpha} / \mathbf{~}^{\circ}\end{array}$ \\
\hline 1 & 2.7 & 380 & 5 \\
\hline 2 & 2.7 & 380 & 10 \\
\hline 3 & 2.7 & 380 & 15 \\
\hline 4 & 2.7 & 380 & 20 \\
\hline 5 & 2.7 & 380 & 25 \\
\hline 6 & 2.7 & 130 & 30 \\
\hline 7 & 2.7 & 180 & 30 \\
\hline 8 & 2.7 & 230 & 30 \\
\hline 9 & 2.7 & 280 & 30 \\
\hline 10 & 2.7 & 330 & 30 \\
\hline 11 & 2.7 & 380 & 30 \\
\hline
\end{tabular}

\subsection{Peel test}

Because the defects of the composite insulator are generally located internal, and it is difficult to detect [20]. In this paper, A device was designed to measure the interfacial adhesive strength between the sheath and core rod, the interfacial adhesive strength can reflect the degree of damage, as shown in Figure 4. Combining with a universal pressure test machine, then the peeling force, displacement and peeling time can be recorded.

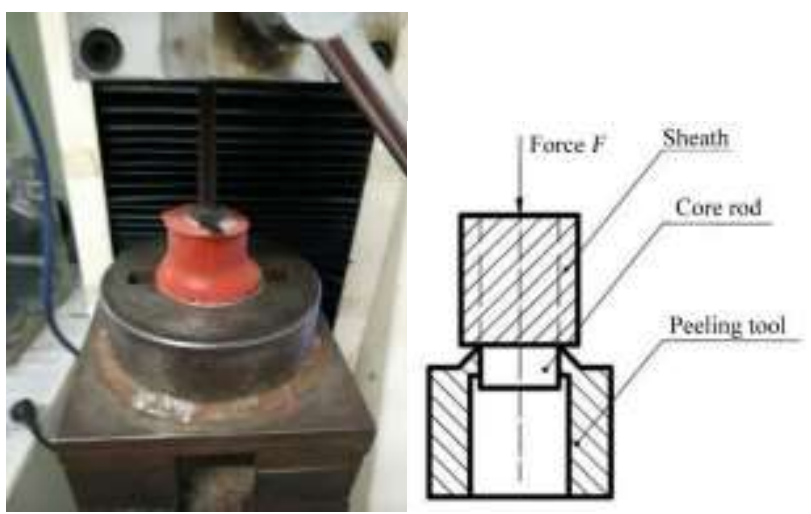

Figure 4. The equipment of peeling the sheath

Take the fatigue test insulators as the specimens. Every insulator is divided into five equal parts, each of which is $200 \mathrm{~mm}$, and then intercept the specimen which the length of sheath is $30 \mathrm{~mm}$, length of the core rod is 50 $\mathrm{mm}$ from each part, and number 1-5, as shown in Figure 3. In order to avoid the influence of radial elastic deformation of the sheath when the sheath is being peeled off, the sheath of the specimen is divided in advance into four equal sections in the circumferential direction. Figure 5 presents the peeling force and displacement curve of interface debonding process. 


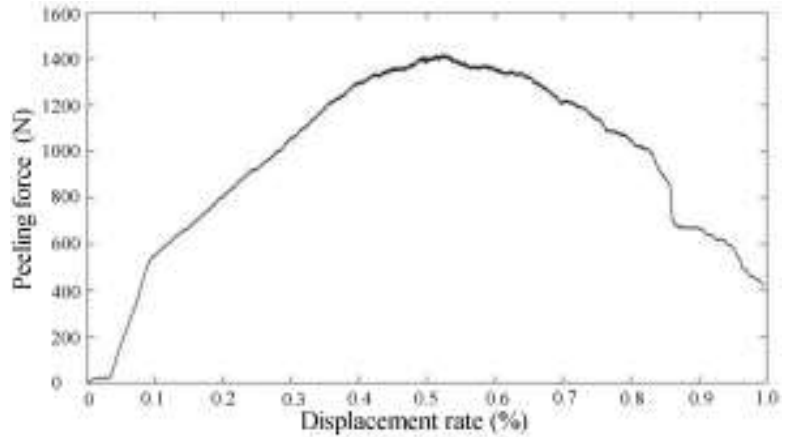

Figure 5. The curve of interface debonding process

\section{Peering mechanics analysis}

The constitutive model of the bonded region established by Needleman et al. describes the continuous relationship between the tensile and the interfacial debonding displacement of unit area, without regard to the nature of the bonding material and the bonded material as a direct influencing factor. The vertical and shear displacements between two points of the material bonding interface can be expressed as:

$$
\left\{\begin{array}{l}
T_{n}=n \cdot \boldsymbol{T} \\
T_{b}=b \cdot \boldsymbol{T} \\
T_{t}=t \cdot \boldsymbol{T}
\end{array}\right.
$$

Where $n, b, t$ are the three directions of the Cartesian coordinate system, $\boldsymbol{T}$ is the unit vector in three direction, and $T_{\mathrm{n}}, T_{\mathrm{b}}, T_{\mathrm{t}}$ are the separation forces normalized for the interface and tangent to the interface at the interface crack tip per unit area.

Zhang [22] thinks that there is a linear relationship between the tension and the displacement along the interface and the tension-displacement along the vertical interface according to the Needleman's interfacial adhesion model. The peeled off the interface crack tip bonding point is taken as point $\mathrm{a}$, and the initial peeling point on the sheath is taken as the b, so these two points along the axial displacement is $u_{\mathrm{b}}$, and displacement perpendicular to the interface is $u_{n}$. When the sheath detached from the core rod completely, the displacement vertical to interface $u_{n}$ set to $\delta$, which represents the interface sticking feature length [21-22].

$$
\left\{\begin{array}{l}
v_{n}=\frac{u_{n}}{\delta} \\
v_{b}=\frac{u_{b}}{\delta} \\
v_{t}=\frac{u_{t}}{\delta}
\end{array}\right.
$$

$$
\begin{array}{ll}
T_{n}=E \cdot F(d) \cdot v_{n} & u_{n} \leq \delta \\
T_{b}=G_{\mathrm{b}} \cdot F(d) \cdot v_{\mathrm{b}} & u_{b} \leq \delta \\
T_{t}=G_{\mathrm{t}} \cdot F(d) \cdot v_{\mathrm{t}} & u_{t} \leq \delta
\end{array}
$$

On basis of the model established by Needleman, Tvergaard [23] limited:

$$
\begin{aligned}
& F(d)=(1-d)^{2} \\
& d=\max \sqrt{v_{n}^{2}+v_{b}^{2}+v_{t}^{2}}
\end{aligned}
$$

The potential energy expression of the bonding interface is as follows:

$$
\phi \operatorname{sep}\left(u_{n}, u_{b}\right)=-\int_{0}^{u}\left[T_{n} d u_{n}+T_{b} d u_{b}\right]
$$

The relationship between the theoretical peeled tensile force and displacement is derived from the constitutive relationship between the viscoelastic body and the bonding interface.

Combined with the tool peeling force model in [24] and the structural characteristics of the composite insulator core rod and sheath, when the interface between sheath and core rod is peeled completely and the tool is wedged into the interface, the inclination of the tool bears partially the pressure $F_{N I}$ of the sheath, friction $F_{f l}$ along the sheath tangential, core rod pressure $F_{N 2}$ on the tool, along the core rod tangential friction $F_{f 2}$. The tool force is shown in Figure 6 (a). Corresponding, the peeling pressure of tool to the bonding interface causes it to separate gradually, and the force is decomposed into an opening force $T_{n}$ perpendicular to the interface and a shear force $T_{b}$ along the interface. The force separated at the crack tip of the bonding interface is shown in Figure 6 (b).

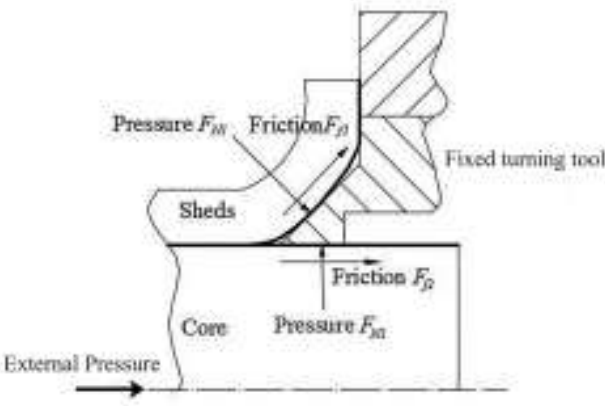

(a) The Tool-stressed model

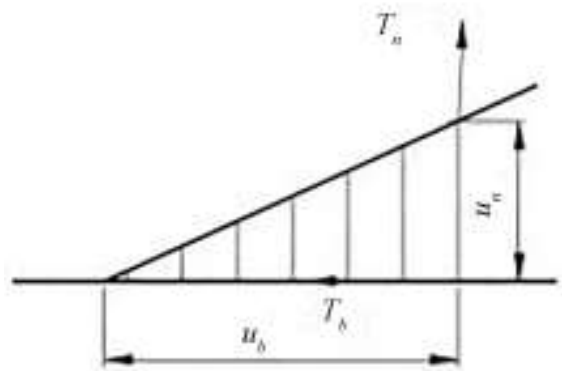

(b) The force separated at the crack tip of the bonding interface

Figure 6. The schematic of the separation of the core/sheath interface

The peel test shows that $u_{n}$ is along the vertical interface, $u_{b}$ is perpendicular to the interface, and the difference between them is very small, so we can assume 
that $u_{n}=u_{b}$. Due to the extrusion of the core rod on the inner wall of the annular tool, the pressure still exists after debonding the sheath completely. According to the formula (3)-(8), the formula is amended as:

$$
T_{\mathrm{b}}=G \cdot\left(v_{b}^{3}-2 \sqrt{2} \cdot v_{b}{ }^{2}+2 v_{b}\right)
$$

According to the stress formula of shear test,

$$
\tau=\frac{F}{S \cdot v_{\mathrm{b}}}
$$

Where $\tau$ is the interfacial shear strength, $F$ is the force from press machine, and $S$ is the bonded interface area of the specimen. Combine formula (9) and (10), get the relationship of pressure shear force and displacement as follows.

$$
F=G \cdot S \cdot\left(v_{b}{ }^{4}-2 \sqrt{2} \cdot v_{b}{ }^{3}+2 \cdot v_{b}{ }^{2}\right)
$$

After fatigue dynamic load test, the interface between housing and core rod of the insulators would occur damage inevitably, which leads to the interfacial bond strength decreased. In order to show the constitutive relation of the interface after the fatigue damage, the coefficient $\chi$, the migration coefficient $\alpha$ and the debonding coefficient $\mu$ are introduced. The coefficient $\chi$ is the degree of pressure drop in the process of peeling and $\alpha$ is the change of the position of maximum peeling force $F_{\max }$, and the debonding coefficient $\mu$ describes the slope of the peeling force and displacement when the interface debonding finally. After the coefficients being corrected, the interface damage model of core rod/sheath considering fatigue influence is obtained as follows:

$$
F=\chi \cdot G \cdot S \cdot\left\{\left(v_{b}+\alpha\right)^{4}-2 \sqrt{2} \cdot\left(v_{b}+\alpha\right)^{3}+\mu \cdot\left(v_{b}+\alpha\right)^{2}\right\}
$$

\section{Results and discussion}

\subsection{Peeling force - displacement relationship}

Here we aim to analyse the damage degree of the adhesive interface of the same position of the different insulator specimens under the situation, that the tensile force and torsion angle in experiment remain constant but swing amplitude is variable. The self-made pressureshear peeling device is used to separate the core rod- sheath interface. The compressive shear device is used to measure the interface quality. The shear forcedisplacement curve was obtained as shown in Figure 7. and the modified theoretical model of interfacial adhesion damage was verified reasonable.

Figure 7 indicates the experimental values curve can reflect the change of interface peel test, but the theoretical values of peel force is lower than the experimental value in the initial stage. The main reason is the material elastic deforms leading to the interface suffer the resistance at this stage and it results in deviation between the actual peel force and theoretical value. Table 2 shows the change of fatigue damage factor under different swing.

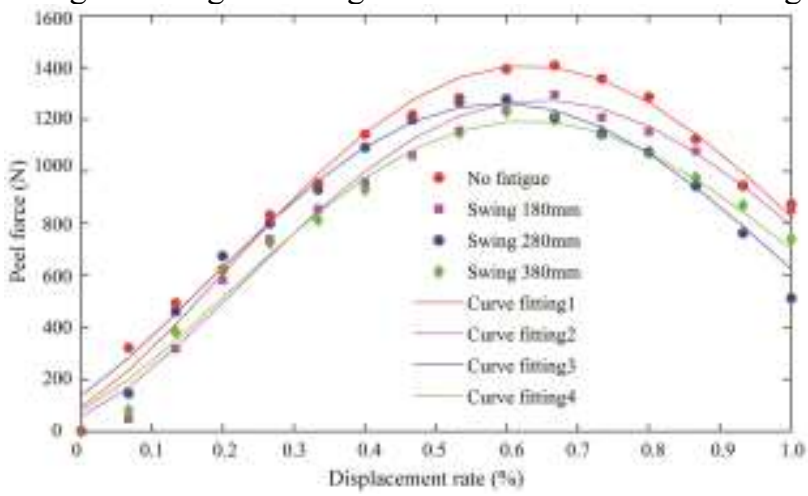

(a) The sampling point No.1

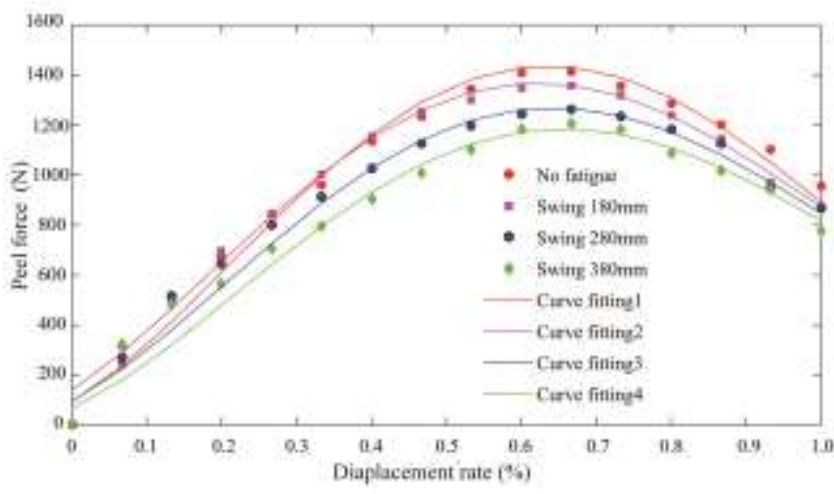

(b) The sampling point No.5

Figure 7. Change curves of peel force with displacement rate

Table 2. Fatigue damage factor under different swing

\begin{tabular}{|c|c|c|c|c|c|c|}
\hline \multirow{2}{*}{$\begin{array}{c}\text { Swing } \\
\text { amplitude } \\
\boldsymbol{L} / \mathbf{m m}\end{array}$} & \multicolumn{3}{|c|}{ Sampling point No.1 } & \multicolumn{3}{c|}{ Sampling point No.5 } \\
\cline { 2 - 7 } & $\begin{array}{c}\text { coefficient } \\
\chi\end{array}$ & $\begin{array}{c}\text { Debonding } \\
\text { coefficient } \boldsymbol{\mu}\end{array}$ & $\begin{array}{c}\text { Migration } \\
\text { coefficient } \boldsymbol{\alpha}\end{array}$ & $\begin{array}{c}\text { coefficient } \\
\chi\end{array}$ & $\begin{array}{c}\text { Debonding } \\
\text { coefficient } \boldsymbol{\mu}\end{array}$ & $\begin{array}{c}\text { Migration } \\
\text { coefficient } \boldsymbol{\alpha}\end{array}$ \\
\hline 0 & 1.302 & 2.03 & 0.1 & 1.302 & 2.03 & 0.1 \\
\hline 180 & 1.191 & 2.03 & 0.08 & 1.192 & 2.07 & 0.13 \\
\hline 280 & 1.179 & 2.03 & 0.13 & 1.105 & 2.07 & 0.11 \\
\hline 380 & 1.105 & 2.03 & 0.1 & 1.031 & 2.07 & 0.095 \\
\hline
\end{tabular}


As a result of the fatigue damage, the overall interface peel force decreases, which has no significant effect on the maximum peeling force $F_{\max }$. As can be seen from Table 2, at specimen part 1, the damage factor $\chi$ decreases from 1.302 at no swing to 1.105 at $380 \mathrm{~mm}$ swing, decreasing by 0.197 . The coefficient $\chi$ at sampling point 5 near the bottom of the insulator decreases from 1.302 without fatigue down to 1.031 at the swing of $380 \mathrm{~mm}$, decreasing by 0.271 . The coefficient $\chi$ changed more obviously on the specimen No. 5, while the displacement coefficient $\alpha$ showed no obvious change, and still fluctuating around 0.1, which means the stress on the core rod/sheath interface near the bottom is greater and the interface damage is more serious. And the change of fatigue conditions has no obvious influence on the location of the maximum of peeling force $F_{\max }$ appears. The locations where the maximum peeling force $F_{\max }$ all lie in about $2 / 3$ of the total displacement on samples.

For No. 1 specimen near the upper metal fitting, When the fatigue load was applied, no matter how the swing changed, the debonding coefficient $\mu$ would not change any more and keep at 2.03. Meanwhile, the specimen No.5 near the lower metal fitting, with the swing amplitude increasing, the coefficient $\mu$ gradually increased, and eventually stay at 2.07 . It presents that the location of the force to the insulator has an important influence on the interface debonding coefficient $\mu$, but the swing amplitude has little effect on it.

\subsection{Interface separation work analysis}

Integrating the load-displacement curve of the compressive shear test and using the unit area separation work that sheath peeled away from the core rod can analyze interface bond strength changes intuitively. Figure 8 presents the unit area separation work of the core $\mathrm{rod} /$ sheath interface in different work conditions obtained by the compressive shear test.

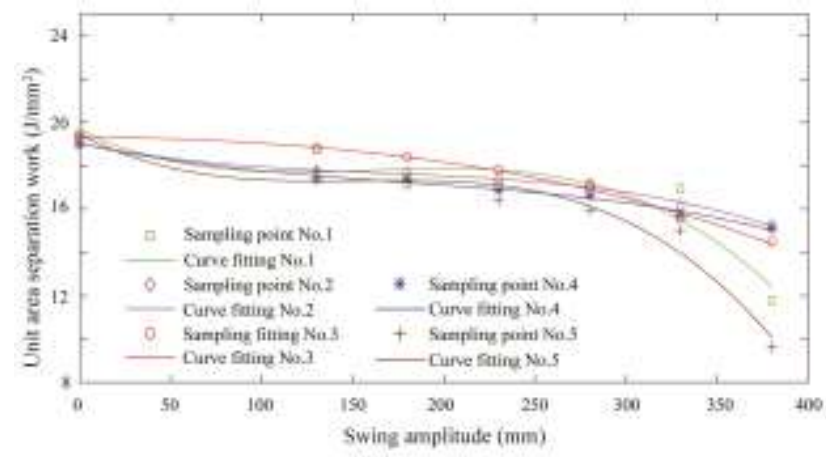

(a) The W- $L$ curve of swing amplitude and interface separation work

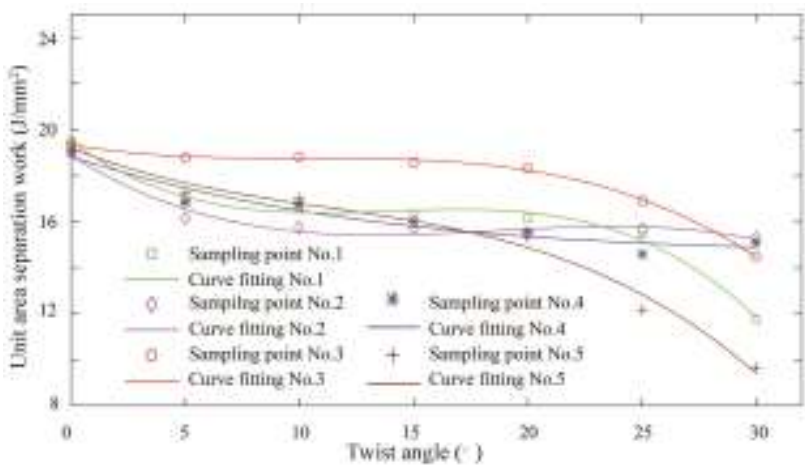

(b) The W- $\alpha$ curve of twist angle and interface separation work Figure 8. Unit area interface separation work of various parts

As shown in Figure 8(a), when the swing is constant, the unit area separation work of all insulator specimens declines the increase of torsion angle. However, the change of the No.2 and No.4 specimens is little and the others decline rapidly once the twist angle is more than 20 degrees. That demonstrates the interface damage of corresponding parts are more serious. As is shown in Figure $8(\mathrm{~b})$, when twisting angle is constant, the unit area separation work of interface of all insulator specimen is decrease with the increase of the swing. However, the change of the No.1 and No.5 specimen is large once the swing is more than about $250 \mathrm{~mm}$ and the decline of others is not obvious which shows the interface damage of the top and bottom parts are more serious. Then we can find the vulnerable locations of the composite insulator in operating conditions.

\subsection{Interface peeling time}

The compressive shear test was carried out by driving the press with constant power. The peeling time shows the sheath can reflect the strength of residual adhesion of the interface after the dynamic fatigue test. Figure 9 shows the time of sheath peeling from different parts of the insulator under the different fatigue parameters.

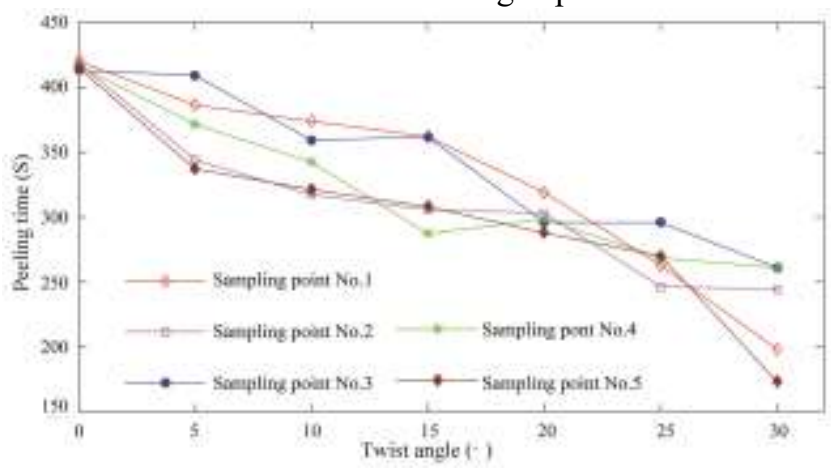

(a) Peeling time of samples in different twist angles 


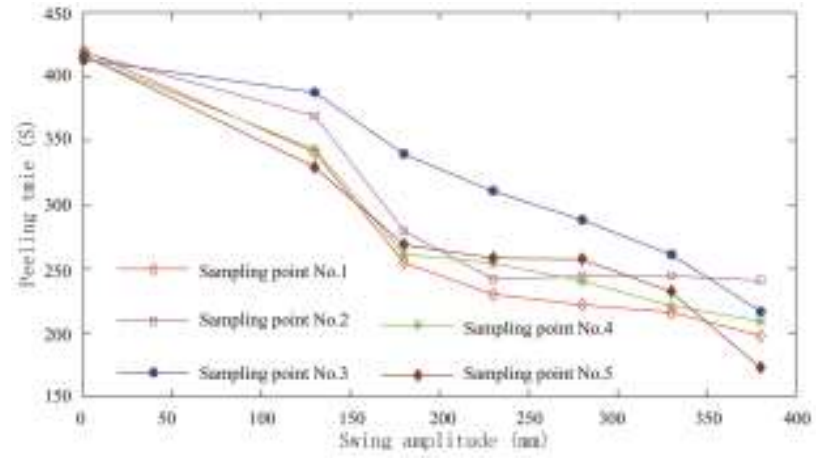

(b) Peeling time of samples in different swings Figure 9. Peeling time of various location samples of the composite insulator

As shown in Figure 9(a), with the twist angle increasing especially with No.1 and No.5 specimens, the peeling time of the sheath away from the core rod is decreasing monotonically. Meanwhile, when the swing amplitude $L$ is a variable, the peeling time of each part is more relevant with swings. For example, when the swing amplitude increases, except the peeling time of the specimen point No.3 which is located in the middle of the insulator decreases linearly, and the other parts go through the trend of firstly is quick then become slow and finally become quick. For the specimens point No.1 and No. 5, which is near two metal fittings, the peeling time declines most obviously. The results show that the change of the swing amplitude has little influence on the interface damage, but the interface near the joint of two metal fittings has serious damage. The change of torsion angle makes more damage to the insulator core $\mathrm{rod} / \mathrm{sheath}$ interface.

\section{Conclusions}

We have successfully modified cohesive interfacial damage constitutive model and used it to characterize the process of core rod/sheath interface of the composite insulators under the different swing amplitudes and twist angles. We designed and made a device of peeling the sheath to analyse the damage degree of the interface.

We studied that the interface damage of the core rod/ sheath when the swing amplitude and the twist angle are taken as the variable respectively. We confirm that the interface damage was affected by swing amplitude and twist angle and twist angles has more serious influence to the core rod/sheath interface than swing amplitude. Also, the peering time and force demonstrate that the damage of the two ends of the insulator near metal fitting is more serious than that in middle of the insulator under the same conditions.

\section{Acknowledgement}

This work was supported by the National Natural Science Foundation of China (No. 51475264 and 51605254); and Natural Science Foundation of Hubei Province (No. 2016CFB565).
1. T. YUAN, R. ZHANG, Q. ZHANG, et al., Insulators and Surge Arresters, 138,19-24+31(2015)

2. Y.F. GAO, X.D. LIANG, Proceedings of the CSEE, 36, 5070-5077(2016)

3. F.Z. ZHANG, L. SONG, R.H. LI, et al., High Voltage Engineering, 38, 3093-3100 (2012)

4. W. HU, C. ZHAO, High Voltage Apparatus, 54, 9397-103 (2018)

5. Q. XIAO, J.S. CAI, F. GAO, et al., Water Resources and Power, 29, 155-157+177 (2011)

6. X.Y. LI, X.D HAN, F.L ZHANG, et al., Insulators and Surge Arresters, 135, 7-13 (2012)

7. Z.S. XIE, M.S. Thesis, Dept. Mechanical Eng, North China Electric Power Univ., Heibei, China, (2012)

8. Z.S. XIE, Z.Q. WANG, Y. CHEN, et al., Transaction of China Electrotechnical Society, 29, 296-302(2014)

9. Y.B. SHAO, M. LU, D.Y. MA, et al., Insulators and Surge Arresters, 139, 34-39 (2016)

10. Q.G. ZHANG, J.B. ZHOU, H.B. XI, et al., High Voltage Engineering, 40, 987 -994 (2014)

11. F.C. LV, J.Q. MA, F.C. WANG, et al., High Voltage Engineering, 40, 3305-3310 (2014)

12. M. YOU, X. ZHENG, Analysis and application of adhesive strength, 23-58. (2009)

13. J. ZHANG, X. CHEN, H. JIA, Journal of Zheng Zhou University (Engineering Science), 27, 48-51 (2006)

14. Z.Y. SU, G. CHEN, Q.F. LI, et al., Power System Technology, 30, 53-57 (2006)

15. Y. CHEN, Y.S. LIU, J. SHEN, et al., Power System Technology, 30, 58-63 (2006)

16. M.M. DUAN, C. WANG, Y P. TU, et al., High Voltage Apparatus, 52,132-137 (2016)

17. F C. SUN, X D. LIANG, Y X. ZHOU, et al., Power System Technology. 26, 33-36 (2002)

18. M.Z. GAO, R. ZHANG, Z.W. ZHANG. High Voltage Engineering, 35,2591-2596. (2009)

19. X.Z. ZHAO, K WANG, Z.C. FU, et al., Dynamic torsional vibration testing machine for the composite insulators, China patent, 104833469B, (2017)

20. Z.L. HE, Ultrasonic detection of internal artificial defects in a composite insulator. South China University of Technology, China, (2013)

21. A. Needleman, Journal of Applied Mechanics, 54, 525 531 (1987)

22. J. ZHANG. Ph.D. dissertation, Dept. Chemical Engineering and Technology, Tianjin University, Tianjin, China, (2005)

23. V. Tvergaard, Material Science, 125, 203-213 (1990)

24. W.J. LIU, M.S. Thesis, Dept. Mechanical manufacturing and automation, Dalian University of Technology, Liaoning, China (2015)

\section{References}

